\title{
Recognition of Opinion Leaders in Micro-Blog based on Linked Data
}

\author{
Zhiyun Zheng, Pengfei Li, Xingjin Zhang, Dun Li* \\ School of Information Engineering,Zhengzhou University, Zhengzhou 450001, China
}

\begin{abstract}
In view of the lack of subjectivity and accuracy in the traditional micro-blog opinion leader recognition method to measure the important factors of users, a new micro-blog opinion leader recognition method is proposed. This paper used the linked data to describe the microblog data, used the association rule mining algorithm to quantitatively determine the important factors that affected the users' ranking, and constructed the opinion leader recognition model according to the index scoring method. Experiments show that our method using linked data identifies the opinion leaders the same as the standard leaders, is more accurate and has better feasibility than that of traditional data.
\end{abstract}

Keywords: linked data; micro-blog; opinion leader; association rule

(Submitted on July 25, 2017; Revised on August 30, 2017; Accepted on September 15, 2017)

(This paper was presented at the Third International Symposium on System and Software Reliability.)

(c) 2017 Totem Publisher, Inc. All rights reserved.

\section{Introduction}

Micro-blog [4] is a social network platform for users to publish, share, distribute and acquire information, and is a kind of innovation to the mode of information diffusion, which plays an important role in the promotion of information. Different from Web1.0's traditional network media, online users become another important source of information in the new Web2.0 media represented by micro-blog. Due to the explosive growth of micro-blog users and the divergence of micro-blog information, micro-blog plays an increasing important role in the formation and development of public opinion. Opinion leaders are the most important media for information dissemination in political election, emergency communication and other social events.

The recognition of micro-blog opinion leaders is the research focus in social networking, but the study is still in its infancy. Massive micro-blog data, complex user relationships and a wide range of data attributes ensure the perfection of the micro-blog functions, but they also bring many obstacles to expression of information accurately. Therefore, we intend to use linked data $[2,3,7]$ to express information and mining association rules, identify key factors, and build micro-blog opinion leader recognition model.

The content of the paper is arranged as follows. Section 2 introduces the related research of the opinion leader recognition. Section 3 establishes micro-blog linked data. Section 4 builds micro-blog opinion leader recognition model based on the association rules mining algorithm. Section 5 obtains micro-blogging data and experimental results analysis. Section 6 summarizes and prospects.

\section{Related Studies}

Opinion Leader was first proposed by the American scholar Lazarsfeld [8]. He believed that information is transmitted according to the mode from media opinion leaders to audience. Opinion leaders are often able to provide information for others in interpersonal communication network, influence people and play an intermediary and filtering role in the mass communication.

\footnotetext{
* Corresponding author.

E-mail address: ielidun@zzu.edu.cn.
} 
At present, the common research on opinion leaders recognition is divided into two categories: social network structure mining and index scoring.

- Social network structure mining methods are generally based on the relationship between users to build social networks, and then use the network structure algorithm to analyze the link relations between users, calculate the importance of the user ranking, and then the front in the sequence is the opinion leader. Weng proposed TwitterRank method of the influential user discovery in the specific topic based on the PageRank [11]. Xiao Yu presented LeaderRank's opinion leader discovery algorithm adding emotion weight in the PageRank [12]. These methods are the improvement of PageRank algorithm and have some limitations in the micro-blog platform.

- Index scoring method is based on the characteristics of opinion leaders. Through the characteristics analysis of the opinion leaders, the corresponding index system were established and marked. Vergani chose three indicators of social identity, expertise and social capital [5]. Ding Hanqing built some indicators including centrality, activity, cohesion, and infectivity [9]. According to the characteristics of micro-blog, Liu Zhiming established a relatively complex index system, including the influence of the two levels of active indicators, in the first level there were two indicators of influence and activity, in the second level there were 7 indicators of the number of repost, the number of comment, the number of mention, the number of original, the number of reply to own blog, the number of reply to others blog and the number of active days [6]. After the establishment of the index system, one method is that they determine the weight through hierarchical analysis [10] and calculate the weighted average to get the final result; another is to construct the scoring model directly and calculate the evaluation function to get the final result. However, the indexes in these methods are based on qualitative analysis of existing research results and lack of objectivity.

In this paper, the association rules mining algorithm and index scoring method are combined to determine the index quantitatively and identify the opinion leaders of micro-blog.

\section{Linking the Micro-blog Data}

With micro-blog's massive data and the complex relations, the linked data is simple and effective. The complete expression of micro-blog data in the semantic information and relations avoids loss of information and redundancy, and provides help for information sharing and other applications. At present, there is no unified, authoritative linked micro-blog data. Drawing on the idea of Seven Stages ontology construction method, this section establishes micro-blog linked data using Protege [10] ontology editing tools in four aspects of the concept, attributes, relationships and examples.

\subsection{Concepts}

Around the micro-blog's attention, point praise, forwarding, comments and other functions, the core contains three aspects such as user, blog and topics. Blogs and topics can be divided into 48 and 45 categories through labels. But these categories will make the blogs and topics classification too similar and too large, not the best classification. If we make the labels as the property of blogs and the topics, it will cause the inconvenience of data input. As a result, we take the labels as the micro-blog concepts and establish the relations between labels and blogs and topics to express explicitly. According to the different ways of certification, users can be divided into four categories: individual authentication users, the official certification users, self-certified users and ordinary users. According to the blog richness, blog can be divided into four categories, which contain pictures blogs, video blogs, pictures and video blogs and pure text blogs.

\subsection{Attributes}

Attributes in linked data are used to describe conceptual properties and relationships. Attributes can be divided into data attributes and object relationship attributes. The data attribute describes the characteristic and basic information of the concept. The object relational attribute describes the relationship between two concepts. In micro-blogs, the user's data attributes include age, gender, location, number of blog posts, educational information, job information, number of friends and number of followers. Blog's data attributes include the number of likes, the number of comments, the number of reposts and time. The data attributes of the topic include the number of discussions and the number of readings. Object relationship attributes include collection, comment, release, friend, follower, inclusion, like, mention, share, similar, create and join. The properties of micro-blog are shown in Figure 1, 2, 3, and 4. 


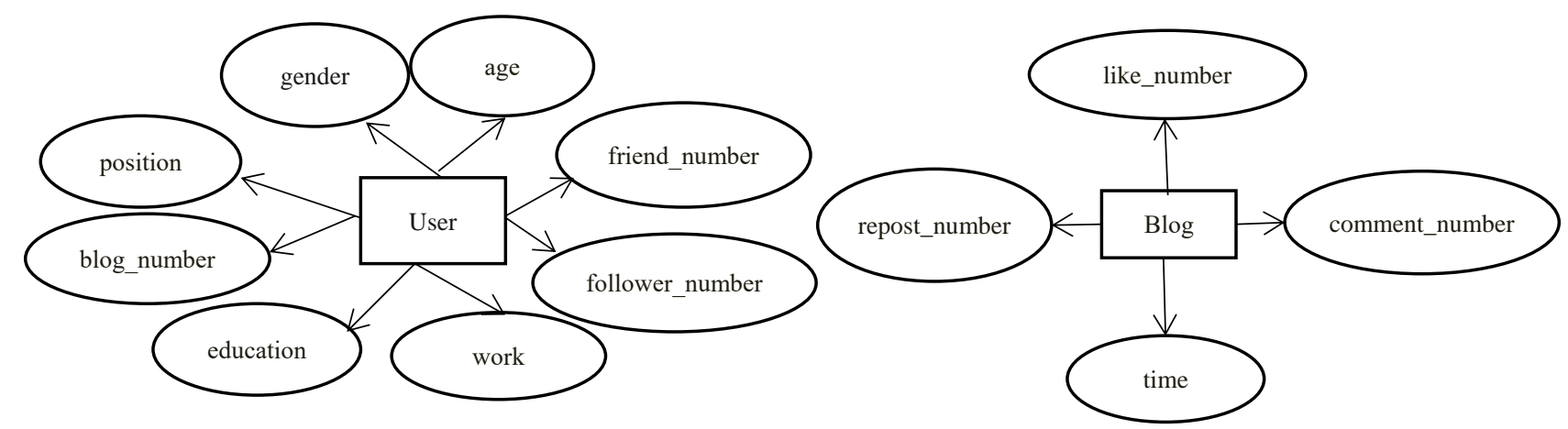

Figure 1. User data attribute graph

Figure 2. Blog data property diagram

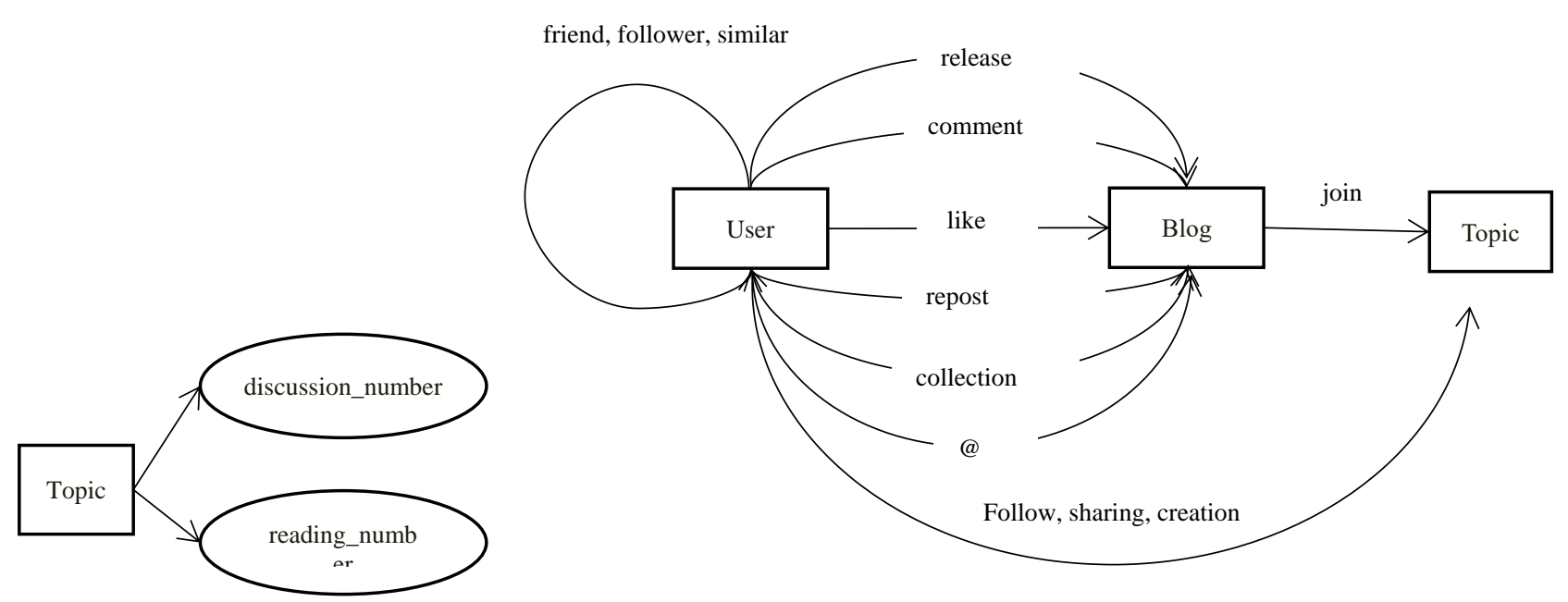

Figure 3. Topic data attribute graph

Figure 4. Micro-blog object relationship attribute map

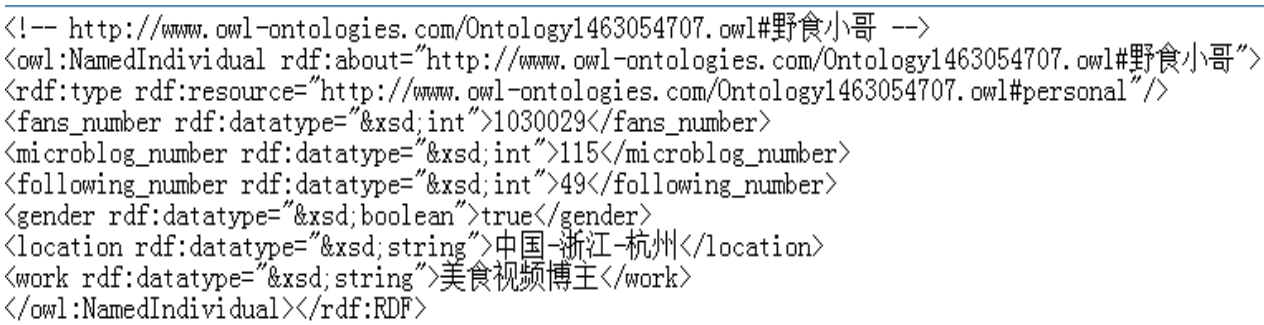

Figure 5. RDF data instance fragment

\subsection{Examples and Associations}

The object class or concept describes the abstract with the same characteristic, data attribute and object attribute description. The object attribute provides the range and type of the attribute, while the instance is a concrete manifestation of the concept. Many instances form a concept. Examples are concrete and clear. An instance has all the attributes of the delegated concept. The instance is concrete and explicit. For example, “野食小哥、男、中国-浙江-杭州、美食视频博主、1030029

、49、115" is a user instance. The instance is the main part of the information and the concrete description which is our direct object of the research. The reasonable instances construction could give the real and effective meaning of the data. The constructed RDF instance fragment is shown in Figure 5.

\section{Opinion Leaders Recognition}

Different to the previous subjective opinion leader recognition model of index factors, we use the linked data and the 
association rule to mine the key factors, and combine with traditional index scoring method to identify opinion leaders of micro-blog.

\subsection{Transaction of Linked Data}

Focusing on RDF data, we extend the SPARQL syntax to define the mining model, generate the corresponding item sets according to the mining model, and the transaction following. Then, the association rules mining algorithm is used to generate the association rules. The mining model consists of two parts: the target concept set and the item sets. The target concept set refers to the set of the concepts that can be related through the relevant attributes in the model. The item set is used to specify the origin of the item set that is a set of attributes. Our mining model is $\mathrm{Q}=($ User, $\{$ Properties $\}$ ), the target concept User represents the micro-blog user, and the item sets concept Properties represents all the attributes in the microblog linked data.

With a series of instances, the project forms the item set and generates a transaction. The instance project comes from the stored information. A fragment of an instance corresponding to the micro-blog linked data is shown in Figure 6.

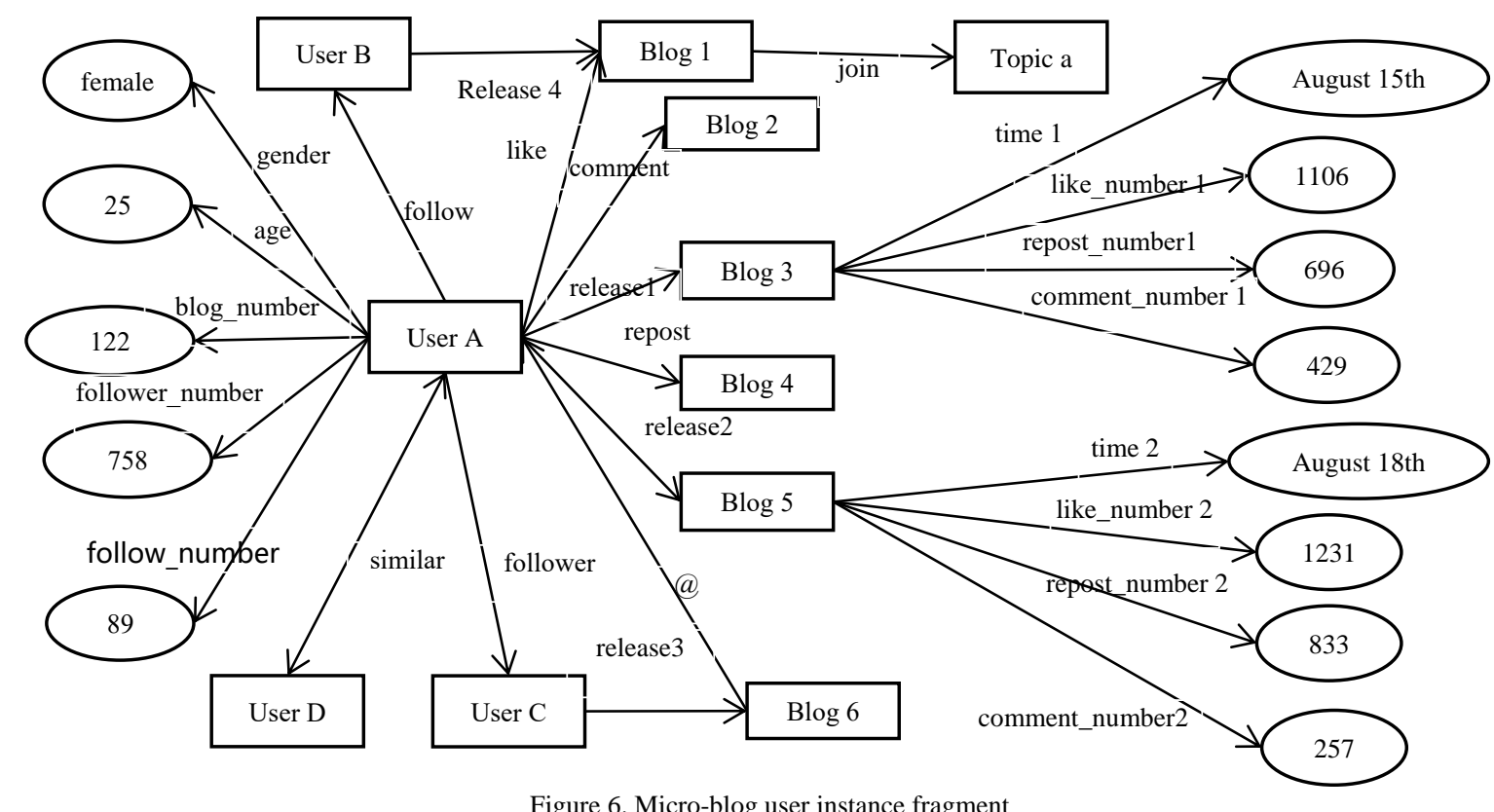

Figure 6. Micro-blog user instance fragment

The instance fragment shown in Figure 6 provides the example fragment to illustrate the process of data conversion. It includes all information about user $A$, such as the basic attributes of the user $\mathrm{A}$, the relevant information of the user and blog that related with the user $A$. In the depth-first algorithm, we start from the user-specified target concept $A$, stop until meeting the user-specified attribute, record the transaction in triple, and then continue in another path.

The information in the graph is converted into triples of subject-path-object. As shown in Table 1, the tuple generation algorithm is described as follows.

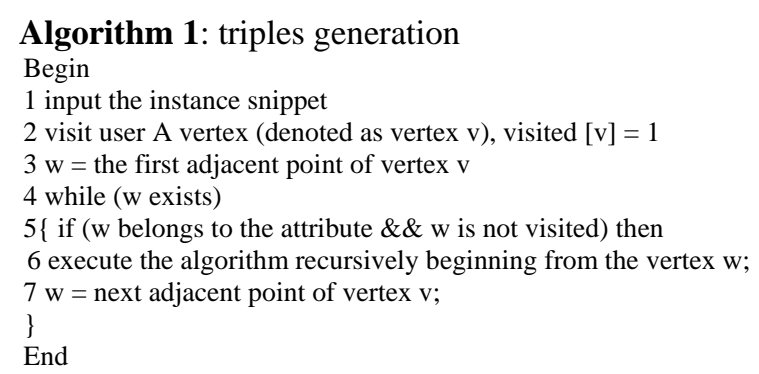


Table 1. The information of example User A

\begin{tabular}{|l|l|l|l|l|}
\hline \multicolumn{1}{|c|}{ ID } & \multicolumn{1}{|c|}{ subject } & \multicolumn{1}{|c|}{ object } & \multicolumn{1}{c|}{ feature } \\
\hline 1 & User A & (follow, release4, join) & Topica & join \\
\hline 2 & User A & (follow, release4) & blog1 & release \\
\hline 3 & User A & (follow) & UserB & follow \\
\hline 4 & User A & (like, join) & Topica & join \\
\hline 5 & User A & (comment) & blog2 & comment \\
\hline 6 & User A & (release1, time1) & August 15th & time \\
\hline 7 & User A & (release1) & blog3 & release \\
\hline 8 & User A & (release1, like_number1) & 1106 & Like_number \\
\hline 9 & User A & (release1, repost_number1) & 696 & repost_number \\
\hline 10 & User A & (release1, comment_number1) & 429 & comment_number \\
\hline 11 & User A & (repost) & blog4 & repost \\
\hline 12 & User A & (release2, time2) & August 18th & time \\
\hline 13 & User A & (release2) & blog5 & release \\
\hline 14 & User A & (release2, like_number2) & 1231 & like_number \\
\hline 15 & User A & (release2, repost_number2) & 833 & repost_number \\
\hline 16 & User A & (release2, comment_number2) & 257 & comment_number \\
\hline 17 & User A & (follower, release3) & blog6 & release \\
\hline 18 & User A & (follower) & UserC & follower \\
\hline 19 & User A & (similar) & UserD & similar \\
\hline 20 & User A & (gender) & female & gender \\
\hline 21 & User A & (age) & 25 & age \\
\hline 22 & User A & (blog_number) & 122 & blog_number \\
\hline 23 & User A & (follower_number) & 758 & follower_number \\
\hline 24 & User A & (follow_number) & 89 & follow_number \\
\hline & & & \\
\hline
\end{tabular}

The triple information in Table 1 produces from Figure 6. The first triple starts from the target user A through the path of follow-release-join, encounters the corresponding value that belongs to the attribute, and then the first triple is generated. The depth-first algorithm is to generate the longest path along the paths, and then generate its sub paths in turn. The second triple is sub-path of the first path and stopped the search until encountering user-specified properties. And so on, 24 triples are generated which forms the initial instance set. In the next step, the final transaction is generated based on the instance set. The process of generating the transaction set from an instance item set is to merge the instance items of the same subpath in the triple according to the attribute set specified by the user, into a transaction. The transactions generated from Table 1 are shown in Table 2.

Table 2. The transaction of instance User A

\begin{tabular}{|c|l|}
\hline ID. & \multicolumn{1}{|c|}{ transaction } \\
\hline 1 & $\{$ follow.release.join.topica,follow.release.blog1,follow.UserB $\}$ \\
\hline 2 & $\{$ like.join.topica $\}$ \\
\hline 3 & $\{$ comment.blog2 \\
\hline 4 & $\{$ release. August 15th,release.blog3,release.like_number $\rightarrow$ 1106,release.repost_number $\rightarrow$ 696,release. comment_number $\rightarrow$ 429\} \\
\hline 5 & $\{$ repost.blog4 $\}$ \\
\hline 6 & $\{$ release. August 18th,release.blog5,release.like_number $\rightarrow$ 1231,release.repost_number $\rightarrow$ 833, release.comment_number $\rightarrow$ 257\} \\
\hline 7 & $\{$ follower.release.blog6,follower.UserC $\}$ \\
\hline 8 & $\{$ similar.UserD\} \\
\hline 9 & $\{$ gender $\rightarrow$ female $\}$ \\
\hline 10 & $\{$ age $\rightarrow 25\}$ \\
\hline 11 & $\{$ blog_number $\rightarrow 122\}$ \\
\hline 12 & $\{$ follower_number $\rightarrow 758\}$ \\
\hline 13 & $\{$ follow_number $\rightarrow 89\}$ \\
\hline
\end{tabular}

In Table 2, the first transaction is the combination of first, second and third triples; the fourth transaction is the combination of the 6th to 10th triples; the sixth transaction is the combination of 12 th to 16 th triples; the seventh transaction is a combination of 17 th, 18 th triples.

\subsection{Data Standardization}

Since the information described in the linked data is very detailed, some data must be pre-processed to the data with statistical characteristics to reflect the relation with the whole, rather than individual's contingency. For example, the microblog number of the user is very large and the number of reviews to every micro-blog must be different, so a micro-blog's data does not illustrate the problem, and we need calculate the average and variance of all of the comments. 
Table 3. Attributes indicators and corresponding methods

\begin{tabular}{|c|c|c|}
\hline Attributes indicators & Processing method & Remark \\
\hline Followers number & Subsection & \\
\hline $\begin{array}{l}\text { Number of authenticated users in } \\
\text { followers }\end{array}$ & Count & \\
\hline Follower interaction rate & Calculate the ratio & $\begin{array}{c}\text { The proportion of followers who participated in } \\
\text { the blog interaction }\end{array}$ \\
\hline Friends number & Subsection & \\
\hline Number of authenticated user in friends & Count & \\
\hline Age & Subsection & \\
\hline Micro-blog frequency & Calculate the frequency & $\begin{array}{l}\text { Calculated by the time and quantity of Micro- } \\
\text { blog }\end{array}$ \\
\hline Number of Micro-blog repost & Calculate the average & $\begin{array}{c}\text { The average number of repost numbers of all blogs } \\
\text { issued by the user }\end{array}$ \\
\hline Number of Micro-blog comment & Calculate the average & $\begin{array}{c}\text { The average number of comment numbers of all } \\
\text { blogs issued by the user }\end{array}$ \\
\hline Number of Micro-blog like & Calculate the average & $\begin{array}{c}\text { The average number of like numbers of all blogs } \\
\text { issued by the user }\end{array}$ \\
\hline Micro-blog richness & Calculate the ratio & Measured in the form of blogs content \\
\hline
\end{tabular}

\subsection{Recognition of Option Leader}

Using the Apriori algorithm [1] generates association rules from the attribute indicators that become opinion leaders, and searches in the frequent item set layer by layer. Firstly, we scan all the transaction records and find the frequent 1-item sets according to the defined support threshold, marked as "M1". And so on, we find frequent $\mathrm{N}$-item sets until we find the largest frequent item sets. At last, association rules are generated and the irrelevant rules are filtered. The rules that meet the requirements are shown in Formula 1.

$$
\mathrm{X} \rightarrow \text { opinion leaders }
$$

Where $\mathrm{X}$ is the index set, the rules in the set are the key indicators to measure the users, and the consequence is the opinion leader. We adopt the Analytic Hierarchy Process (AHP) to assign weight of each indicator: input the user data, mark users' attributes with indicator scoring method, calculate the weighted average, and recognize the opinion leaders. Algorithm 2 is shown below.

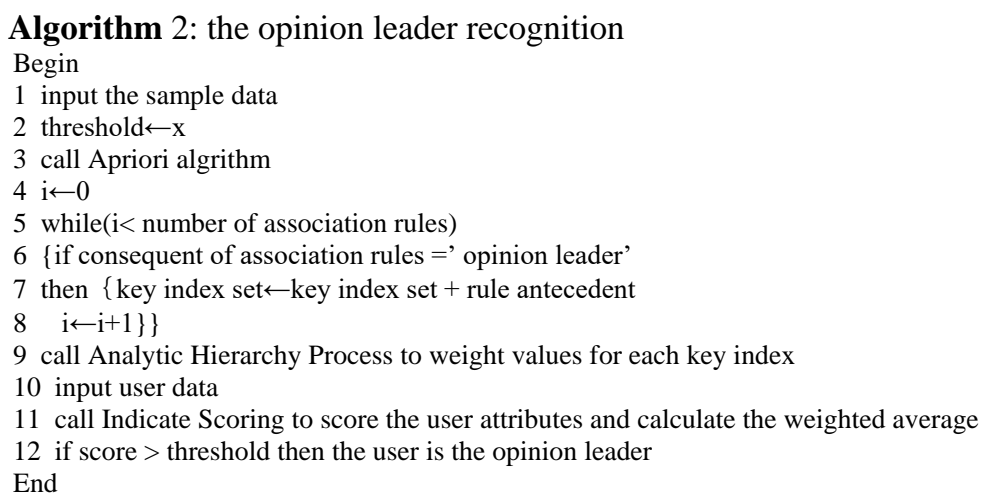

The efficiency of the algorithm is decided by the Apriori algorithm. We analysis the algorithm and get the computation complexity of Apriori algorithm including 3 parts:

a) The production of the frequent itemsets is $\mathrm{O}(\mathrm{Nw})$, where $\mathrm{N}$ is the number of the transactions and $\mathrm{w}$ is the average width of the transcations.

b) The production of the candidates is $\mathrm{O}\left(C_{k=2}^{w} \mathrm{k}(\mathrm{k}-2) C_{k}\right)$, where $\mathrm{k}$ is the item number in the frequent itemsets.

c) The calculation of the support is $\mathrm{O}\left(N_{k} C_{w}^{k} \alpha_{k}\right)$, where the transaction with $|\mathrm{t}|$ length could produce $C_{t}^{k} \mathrm{k}$-itemsets, and $\alpha_{k}$ is the consuming of support count in updating the candidate $\mathrm{k}$-itemset in the Hash tree.

\section{Experiments and Results Analysis}

The experimental environment is Jena 2.6, Eclipse, the hardware configuration is Intel (R), Core (TM), i3-2100, @3.10G Hertz CPU, 4GB memory. The experimental data come from Sina Microblogs. The raw data transform into experimental 
data in two stages of acquisition and screening. In the acquisition stage, Octopus is used to obtain the user's background information and interactive information between users. In the filter phase, the invalid data are deleted. There are 2000 users including a total of 593,909 attribute information. The core flow chart of the experiment is shown in Figure 7.

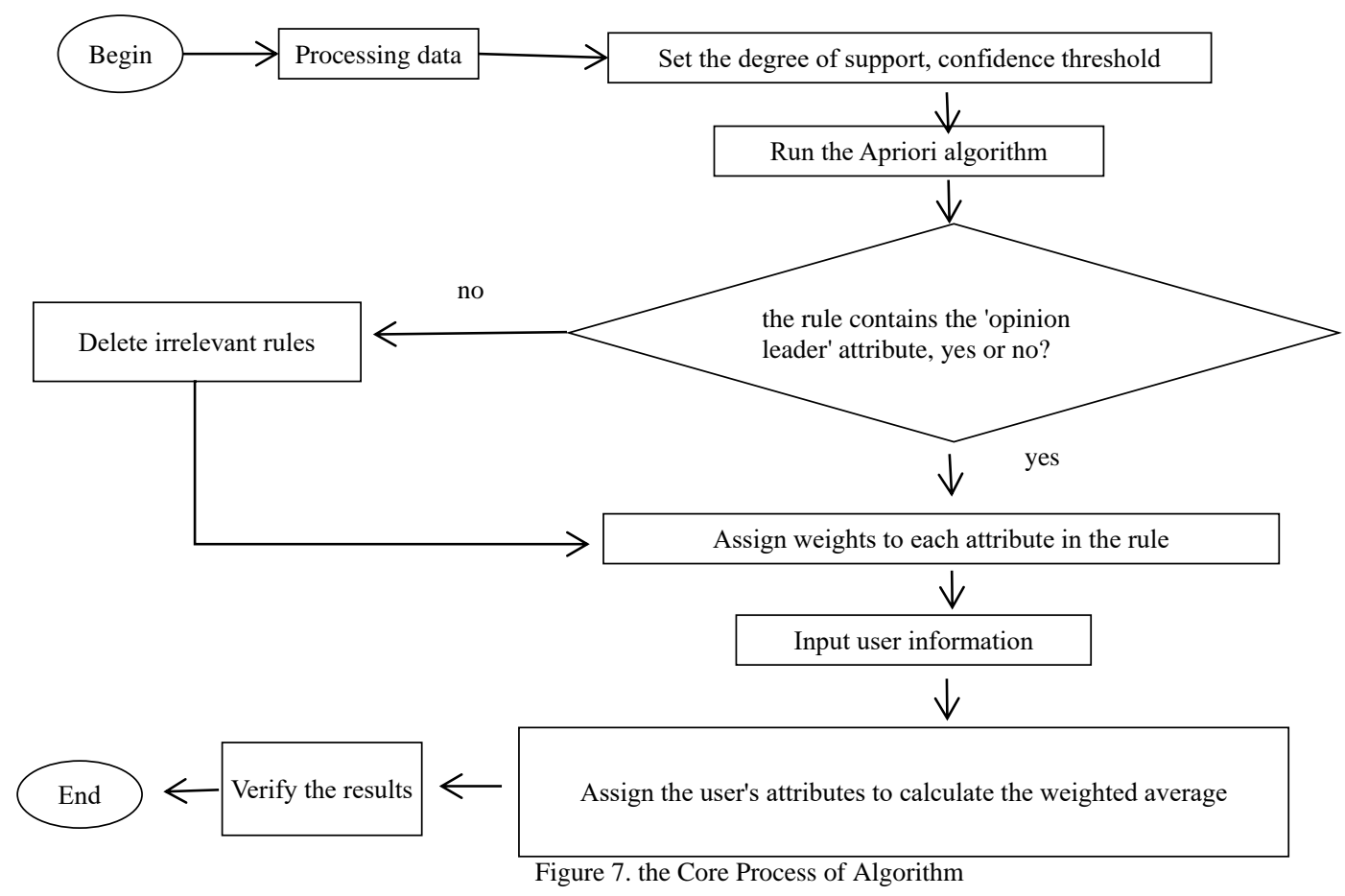

The key indexes and their weights are shown in Table 4.

Table 4. Key indexes and weights

\begin{tabular}{|c|c|}
\hline index & weight \\
\hline Follower_number & 0.2175 \\
\hline Number of authenticated users in followers & 0.1537 \\
\hline users' category & 0.1678 \\
\hline Follower interaction rate & 0.1460 \\
\hline Number of Micro-blog repost & 0.1372 \\
\hline Number of Micro-blog comment & 0.0889 \\
\hline Number of Micro-blog like & 0.0889 \\
\hline
\end{tabular}

The Micro-blog Fengyun list (Hall of Fame) is user value and data services platform authorized by Sina micro-blog officials. This platform uses a large distributed system to store, update, and analyse micro-blog data, and adopts a set of complex evaluation algorithms to measure user value and influence. We use the data and rankings provided by the microblog Fengyun list as the experimental evaluation criteria and comparison. The top ten opinion leaders identified in the paper are shown in Table 5.

Table 5. Ranking of opinion leaders

\begin{tabular}{|c|c|c|}
\hline User's Nickname & Our ranking & Fengyun List ranking \\
\hline 头条新闻 & 1 & 1 \\
\hline 央视新闻 & 2 & 5 \\
\hline 人民日报 & 3 & 2 \\
\hline 何炅 & 4 & 6 \\
\hline 新浪娱乐 & 5 & 7 \\
\hline 微博搞笑排行榜 & 6 & 3 \\
\hline 李开复 & 7 & 8 \\
\hline 南方周末 & 8 & 9 \\
\hline 当时我就震惊了 & 9 & 10 \\
\hline 头条新闻 & 10 & \\
\hline
\end{tabular}


As shown in Table 5, the two ranking orders are nearly the same. Among them, there are 9 authentication users and 1 non-authentication user. That shows that certification has a certain impact on the user to become opinion leaders, but not entirely decisive role. Among the top-10, there are 5 individual users, 5 official users, and the order is basically consistent with the micro-blog Fengyun List. Only two users's rank declined, 何炅 and 李开复. The top-10 contains 3 public Figures recognized by the masses of the national and authoritative users because the dissemination of information has certain influence. Other non-public Figures, the majority of users of authoritative media, indicate the masses for the confidence of the official platform. The experimental results show the feasibility of the opinion leader recognition method that based on the associated data.

\section{Conclusions}

Aiming at subjective selection index and the lack of objectivity in the option leader recognition of the traditional method, we constructed Micro-blog linked data and proposed an opinion leader mining method based on the associated data. Firstly, the depth first algorithm is used to pre-process the linked data. Secondly, we used the Apriori algorithm to select the objective influencing factors. Finally, we combined with the index scoring method to recognize the opinion leaders. Experiment shows that the opinion leaders recognized in the paper are nearly consistent with the standard leaders, which is more accurate than the traditional data and possesses feasibility and accuracy.

\section{Acknowledgments}

The authors are grateful to the editors and reviewers for their helpful comments and suggestions. This work was partially supported by National Social Science Foundation project (17BXW065), Science and Technology Research project of Henan province (14430051007) and Science and Technology Research project of Zhengzhou(141PPTGG368).

\section{References}

1. T. Bernerslee, J. Hendler, O. Lassila. "The Semantic Web," Scientific American, vol. 284, no. 5, pp. 34-43, 2001.

2. C. Bizer, T. Heath, T. L. Berners. "Linked Data-the Story so Far". Semantic Services, Interoperability and Web Applications: Emerging Concepts, pp. 205-227, 2009.

3. “China Internet Development Statistics Report”. http://www.cnnic.net.cn/gywm/xwzx/rdxw/201708/t20170804_69449.htm, Last accessed on August 4, 2017.

4. H. Q. Ding, Y. P. Wang. “Analysis of Opinion Leaders Characteristics in SNS Cyberspace: a Case Study of Douban.com”. Journalism \& Communication, no. 3, pp. 82-91, 2010

5. J.Y. Guo, Z. B. Zhang, Q. Y. Sun. "Study and Applications of Analytic Hierarchy Process". China Safety Science Journal (CSSJ), no. 5, pp. 26, 2008.

6. "Linked data", Available at https://www.w3.org/DesignIssues/LinkedData.zh-cn.html, last accessed on September 23, 2017

7. P. F. Lazarsfeld, B Berelson, H Gaudet. "The Peoples' Choice: How the Voter Makes up His Mind in a Presidential Campaign". 1968.

8. Z. M. Liu, L. Liu. "Identification and Analysis of Opinion Leaders in Internet Public Opinion”. Journal of Systems Engineering, vol. 29, no. 6, pp. 8-16, 2011.

9. “protégé". Available at https://protege.stanford.edu/, last accessed on September 23, 2017

10. M. Vergani. “Are Party Activists Potential Opinion Leaders?”. Javnost-The Public, vol. 18, no. 3, pp. 71-82, 2011.

11. J. Weng, E. Lim, J. Jiang, and Q. He, "TwitterRank: Finding Topic-sensitive Influential Twitters," in Proceedings of the Third ACM International Conference on Web Search and Data Mining, 2010.

12. Y. Xiao, W. Xu, L. Xia. "A Network Group Opinion Leader Recognition Algorithm Based on Emotional Tendency Analysis". Computer Science, vol. 39, no. 2, pp. 34-37, 2012. 